\title{
Melanesian tok stori in leadership development: Ontological and relational implications for donor-funded programmes in the Western Pacific
}

\author{
Kabini Sanga \\ Victoria University of Wellington, New Zealand: kabini.sanga@vuw.ac.nz \\ Martyn Reynolds \\ Victoria University of Wellington, New Zealand: msdfreynolds@gmail.com
}

\begin{abstract}
Donor-funded programmes in areas such as leadership development take place in every continent. In the Western Pacific, Melanesia has been host to such programmes based on non-Melanesian thought and practice over the years. However, a review of donor-funded leadership programmes in the region reveals a history of concern regarding effectiveness but no significant change in programme orientation. This article provides a counter-story of a donor-funded leadership programme which utilizes a readily available cultural model of thought and practice of Indigenous origin: tok stori. Tok stori is a form of discursive group communication which is an everyday occurrence in Melanesia. The experiences of leadership mentors operating in $a$ tok stori-centred leadership development programme located in the Solomon Islands provide an opportunity to explore and evaluate what cultural wisdom can contribute as the core of a leadership development programme. The benefits are many: leaders benefit when complex contextual matters can be introduced into leadership development by the openness of tok stori; depth of engagement is supported by the development of mentor-leader relationality as an integral part of the tok stori process; and mentors gain increased expertise in using a cultural form with which they are already familiar in new pedagogical contexts. Ultimately, this is a story of the value of honouring important aspects of culture for those who inhabit the ontology which gives them significance. Re-negotiating the way the cultures of donors and recipients are regarded by programme developers is an important factor in the centring of Indigenous thinking and practices such as tok stori in leadership and other person-centred programmes. The lesson of this article is that there are gains available to all where this occurs.
\end{abstract}

Keywords: tok stori; leadership development; postcolonial theory

\section{INTRODUCTION}

Donor-funded programmes in areas such as leadership development are no doubt wellmeaning. They assume importance when the relationship between the quality of 
leadership and outcomes in areas such as education is considered. However, where ideas from one place are used in another, problems can arise (Fernandez, 2016). In inter-cultural contexts such as donor-funded programmes rolled out in Melanesia, issues which have ontological origin can be provoked by relational inequity between programme funders and programme recipients. This article addresses the area of donor-funded leadership development by examining the case of tok stori, a Melanesian cultural form used as the basis of a programme of leadership development in the Solomon Islands. A programme which uses Indigenous thought and practice as a navigation bucks the trend in donorfunded practice, giving a window on what is possible when re-thinking occurs. An examination of the effects and effectiveness of tok stori in this context provides a counterstory based on Indigenous capital, a kind of community capital (Yosso, 2005) which widens the scope of what could be taken into account when programmes are constructed and evaluated. The story offers an example capable of supporting further developments in donor practice, donor-recipient inter-cultural relations, and in the way Indigenous practices, such as tok stori, are understood in non-traditional contexts. For while tok stori is most relevant in the Western Pacific, ground-breaking developments in this region offer wisdom to the wider world.

\section{Tok stori}

Tok stori, a form of discursive group communication, is an everyday occurrence in Melanesia. As a habitual activity, those who practice may not examine its parameters or dwell on its significance. To an observer, however, its significance is indicated by its ubiquity and flexibility; its parameters shift to suit the needs of Melanesian life. To speak of tok stori is to invoke a way of negotiating with the social world. In tok stori, relationality, information and time come together to form a distinct way of being.

In the literature, some attempt has been made to clarify tok stori as a cultural form. Tok stori has variously been described as an accepted Melanesian way of sharing what is known (Vella \& Maebuta, 2018), an inter-generationally legitimized Melanesian discussion mode (Brigg, Chadwick, Griggers, Murdock, \& Vienings, 2015) and a traditional dialogic problem-solving method based on reflective oral activity (Evans, Guy, Honan, Paraide, \& Muspratt, 2010). These descriptions are linked by evoking an oral activity focused on purposeful sharing and which has a long heritage. In tok stori, storying is something one does together: a story is constructed by speakers and listeners. Tok stori takes place when people interchange and exchange, creating a collective experience in which the development of relationships is both an accompaniment to, and a purpose of, storying. Group activity which develops knowledge is the stuff of life in Melanesian societies: this truth is embodied in the everyday nature of tok stori.

\section{Tok stori, talanoa and context}

Depending on one's perspective, the relationship between tok stori from the Western Pacific and talanoa from the Eastern Pacific may appear distant or proximal. In some contexts, a clear separation has been made. Halapua, a Tongan, deliberately employed the terms talanoa and tok stori when working for inter-group reconciliation following armed conflict in Fiji and the Solomon Islands respectively (Halapua, 2000; Spence \& Wielders, 2006; Talanoa \& Development Project, 2005). A salient aspect in these endeavours was local ownership of process, established by using both a relevant and familiar term, and by framing discursive activity through recognized cultural protocols (Talanoa \& 
Development Project, 2005). Talanoa and tok stori may also appear distinctive in traditional contexts. At the village level, what can even be considered tok stori demands detailed specific attention (Van Heekeren, 2014). Similarly contextual are the levels of talanoa described in Tongan scholarship (Fa'avae, Jones, \& Manu'atu, 2016; Vaioleti, 2013). Thus, in some circumstances, tok stori and talanoa as practiced may seem distantly related. However, if an ontological lens is applied, tok stori and talanoa may appear close.

An ontological lens applied to tok stori and talanoa pays attention to understanding the world as dialogic, relational and processual. Ontologically, tok stori and talanoa may appear overlapping: both are group-based oral negotiations aimed at increased understanding promoted by relational development. This similarity is reflected in the way some writers have used the terms interchangeably (Houma, 2011; Nanau, 2014; Sanga, 2016). A unifying ontological perspective is perhaps most relevant in contexts where connections between people focus on an aspect of non-traditional and/or professional life, such as educational leadership development. Under such circumstances, less prescription or expectation about precise forms of conduct may exist. Instead, what can navigate conduct is the general way one makes sense of the world.

Despite similarities which may sometimes stretch to the point of synonymity, we advocate for tok stori as the appropriate name to use for discursive activity in leadership development in Melanesia. A name can affect the relationship which people understand to exist between themselves and a thing or practice. In Melanesia, the term talanoa may evoke something which needs to be learned or borrowed. Tok stori, however, is known, owned, and can be developed confidently by Melanesians because of their familiarity with it. Thus, in discursive pedagogical leadership development, talking of tok stori places power in the hands of Melanesian leaders as experts. This, in turn, can influence the level of pedagogical effectiveness.

Unfortunately, despite its availability, ubiquity and cultural value, tok stori as a mode of learning is not always used in educational leadership development in Melanesia. Many branches of leadership development have developed their own conceptual languages, ways of operating and implied, if not conscious, understandings of the world. Instead of tok stori, traditions which are more didactic and less deliberately relational are common in the Melanesian region (Sanga, 2009). Examples in educational research include Malasa (2007), who used interviews to study the effectiveness of Solomon Islands school leaders, Akao (2008), who used interviews with Solomon Islands women leaders, and Sisiolo (2010) who used interviews to study school leaders in the Choiseul Province. A common feature of these studies is their deficit-oriented conclusions about Solomon Islands educational leadership. This unquestioned use of non-Melanesian introduced approaches can contribute to a learning environment where the Melanesian mind is a relative stranger and the effectiveness of deliberate leadership development is muted.

\section{Leadership programmes and Melanesian culture}

Leadership initiatives have a long track record in Melanesia. Sanga (2009) reviews two programmes designed to increase leadership capacity among positional leaders in the public service in Solomon Islands. He notes that, although both programmes reflected contemporary environments for reform and dealt with appropriate content and topics, participants, donors and programme deliverers identified issues of effectiveness. He laments a recurrent historical pattern: introduced leadership programmes have frequently 
delivered the same skills, knowledge and competences through Western-devised approaches despite negative experiences of post-training implementation. In this situation, Sanga's answer is context: when devising methodologies, pay attention to the Melanesian mind.

Sanga (2009) describes the Melanesian mind in the context of public service as having three masters: culture (or kastom), church and formalized institutions. Each domain is legitimate, demands allegiance, and competes for loyalty with the others. Programmes which target the second and/or third domain at the expense of the first can result in the misreading of default Melanesian behaviour. Leadership programmes in which linkages and relationships between domains are erased do not well serve the complexities of leaders' lived experiences. To Sanga, "The need is for programme designers to appreciate better, the tensions between understanding of roles, rules and knowledge in [the various] domain[s]" (p. 1). Instead of methodologies which sit well in an alien ontology, collaboration between donors, providers and leaders may offer a pathway to more productive leadership training. A Melanesian mode of collaboration suitable for pedagogic use in the leadership context is tok stori.

\section{RESEARCH: TOK STORI IN LEADERSHIP DEVELOPMENT: A SOLOMON ISLAND EXAMPLE}

We now turn attention to original data regarding tok stori as a pedagogical tool in leadership development in Melanesia. This discussion uses data from a mentorship programme of the Fellowship of Faithful Mentors (FFM) which was aimed at socializing Solomon Islands mentors to support Solomon Islands school leaders in a donor-funded leadership programme which took place in 2017 in the Solomon Islands. The programme had goals for developing leadership capacity in school leaders, generally principals. Commonly, leadership development programmes in Pacific Islands regions use approaches which begin with an assumed needed competence (knowledge, skills). In this Solomon Islands programme, an early programme proposal involved rubrics for semistructured interviews between leadership mentors and practicing leaders. For instance:

1. What is your school vision? 2. Do you have structures and routines in place to support your leadership goals? If so what are they - if not why is this difficult?

These rubrics were designed to scaffold interaction so as to collect data directly relevant to pre-set programme outcomes, such as:

Teachers and school leaders have knowledge of patterns of teaching and learning practice related to instructional learning for their schools.

While outcomes of this nature are defensible as part of a school leadership strategy, the proposed modes of data generation (semi-structured interview), and consequent mentoring against pre-developed targets serve to position power overwhelmingly in the hands of programme mentors or deliverers. However, through consultation, the programme details were reviewed and replaced by a person-development strategy centred on tok stori.

As a tool to review the programme, Sanga (2017) offered a framework for leadership development in Melanesia. This aligns a specific model of leadership with an understanding of the potential of tok stori in leadership development. The leadership 
model draws its inspiration from sources such as Sanga and Walker (2005). It involves five key concepts: the leader as a person; leading as an activity; leadership as the alignment of people, resources, context etc. with a vision towards change; a reference point or True North (George, 2007) by which the leader navigates; and leadership development, movement or change. Between these concepts is a sense-making actionoriented holistic space. In this space, productive intersections of people, ideas, actions and vision occur. For instance, the understanding of leadership held by a leader can generate action, framed through their True North and directed at their vision of change (George, 2007; George, Sims, Mclean, \& Mayer, 2007).

Sanga (2017) aligns this leadership model with an understanding of tok stori as a discursive sharing and investigation of experience, capable of pedagogical or person development. Experientially, leadership is positioned by and between three broad dimensions: the leader; leading or leadership; and the environment. In the Melanesian context, a complex aspect of the environment is inter-domain positionality. This can be appreciated through Houma's (2008) helpful metaphorical 'Iora framework which imagines education as a journey, and school as a canoe in which seats are delegated by the community to various community members. School leadership is "a form of collaborative leadership where authority is shared amongst a team of leaders who head the family groupings which occupy the seats within [a] canoe" (p. 3). Although everyone in the canoe takes part in the journey and thus has leadership responsibilities, who a leader is and where they are sitting affects their experience of leadership. The seat one occupies in the institutional domain of school may not be the same as that one occupies in other domains. Thus, intersections between the person of the leader, their ideas of leadership and their environment shape lived experience. This complexity becomes open to reflection when it is the matter of tok stori.

Where tok stori is used for pedagogical purposes, part of the relational dynamic is the bringing together of lived experiences of leadership and models of how leadership can be thought about. This dynamic sits in the tok stori relational space between leader and mentor. As leaders tok stori their experiences, mentors sensitized to the five key concepts of leadership and cognisant of context can help guide a pedagogical path towards deliberate leadership development. Tok stori conducted within this framework promotes a holistic mentorship which admits all three domains of the Melanesian mind. It does not place theory above experience, nor does it place the mentor above the leader. Instead, embedding leadership development in tok stori honours a leader's Melanesian experience and their environmentally socialized Melanesian mind.

\section{METHODOLOGY}

After the leadership programme had been reviewed, FFM mentors were trained according to the model of leadership discussed above, and the revised tok stori-centred pedagogical navigation was made the core of mentor-leader interaction. Following their experience of tok stori within the programme, seven Melanesian leadership mentors were asked about their experience of the potential of tok stori as a pedagogical tool for people development. The data presented below is drawn from their reflections and was provided in response to three emailed questions and subsequent discussion.

1. How relevant for educational leadership in the Solomon Islands is the view that leadership can be seen in the tok stori of leaders? 
This question interrogates the proposition that tok stori gives status to the experiential expertise of those involved. The question provides an opportunity to reflect on the way the approach legitimizes, values and exposes what is known by leaders about leadership, and addresses foundational matters upon which future leadership can develop.

2. How effective is tok stori as a pedagogy for Solomon Islands educational leaders?

The second question addresses the contextual appropriateness of the methodology in terms of the aims of leadership programmes. It provides an opportunity to reflect on the way using tok stori for leadership development structures a space for learning as well as discussion. This is an evaluative question.

3. How has your use of tok stori (philosophy, application etc.) in a Solomon Island leadership development programme impacted your own

thinking/practice/understandings?

This third and final question addresses the potential of tok stori as a reciprocal experience by asking mentors about their own learning. The phrasing of the question includes alternatives to indicate the breadth of responses possible. This question provides processual opportunities for self-reflection.

Answers to all three questions were collated and thematically analysed. Purposeful sampling ensured the relevance of responses and guaranteed that, despite the small size of the sample, rich data emerged. A question-by-question analysis of the data is presented below.

\section{ANALYSIS}

\section{Question 1: Issues of relevance}

In answer to this questions, two issues of relevance are discussed.

\section{Cultural relevance}

Mentors understand tok stori as a culturally relevant form of social interaction within educational leadership development programmes in a Solomon Island context. A key aspect of this is the congruence between the oral nature of the form and what one participant called a culture of oral tradition of which it is a part. This alignment reflects the descriptions of tok stori and its place in wider Melanesian culture in the literature. Mentors counterpoint the oral focus and written literacy, the focus of education in the West, in three contextually significant ways: as preference, through skill and in time.

The oral focus which underpins tok stori is significant in a Melanesian leadership development context because of the choice-making of practicing leaders when in their own spaces:

[T]oday's school leaders, they prefer talking and chatting to inform you about what they are doing rather than writing and producing reports.

In a context where engagement of Solomon Islander leaders is central, the mentor's response suggests tok stori as a positive way forward. A people development programme which meets participants on their own terms is likely to succeed, especially where prior experiences are legitimized as giving rise to expertise. Because in Melanesia, the cultural 
value of oral language is higher than for written language, writing as a main pedagogical tool may not play to the strengths of leaders whose practice in oral communication has been under daily development for a long time. Tok stori, however, offers leaders an opportunity to use a preferred mode of communication and being as a basis of their own leadership development. Reflecting people's preference in methodology adds to a programme's pedagogical potential.

Tok stori, as a cultural activity, requires oral skills. Although high level writing skills are possible (and desirable and valuable) for school leaders, excellence in oracy is essential in Melanesia. Mentors recognize the high levels of oral skill possessed by leaders:

In the Solomon Islands context, a lot of our leaders are very good at oratory skills. This is not belittling their writing skills but in the context of appreciating our oral traditions in Solomon Islands.

Tok stori can leverage these skills by creating an interactive relational space for oral interaction capable of delivering ideas and developing understanding. Where a leader's preferences and high oral skill level combine with a methodological approach, tok stori as a culturally relevant form for use in leadership development in Melanesia is validated.

Mentors understand tok stori as continuing to have contemporary cultural relevance in changing and turbulent times. This is despite the recognition that some oral traditions may gradually face displacement by writing. One mentor made the case for the temporal significance of tok stori thus:

[O]urs is still a culture of oral communication. Thus, tok stori will remain an essential aspect of people's lives. Our transition into a writing culture will take a while before our society becomes a fully written society.

Tok stori in leadership development recognizes the oral strength of Melanesian cultures in the here and now. It "meets the people" where and when they are and offers an alternative to the priorities of other cultural traditions.

\section{Personal relevance}

Mentors understand that personal relevance contributes to the usefulness of tok stori as pedagogy. Because it is ubiquitous in the Pacific region, mentors socialized into Melanesian practices bring skills and familiarity which are valuable to tok stori as a mode of leadership development in leadership programmes. One mentor observed:

[T]ok stori is something I am familiar with. Culturally, I use tok stori with family and relatives, every day, in the village.

Leadership development can benefit from the kinds of familiar family and village relationality implied in tok stori. An expectation of relational closeness can build a sense of shared purpose and mutual commitment, helpful in the mentor-leader relationship. This is important because the effectiveness of a relational pedagogy such as tok stori can be indicated by the intensity of relatedness experienced by those involved. This is possible where mentors see tok stori as personally relevant.

Mentors also recognize that a leader's personal familiarity with tok stori supports its relevance as a leadership development activity. Familiarity creates a sense of freedom, while the formality of interview, for instance, can restrict what leaders understand to be relevant. 
If allowed to speak with freedom and without intimidation in any form, then Solomon

Islanders will speak comprehensively and cover a wide area in their storying.

Familiarity with tok stori as a practice encourages leaders to bring what is personally relevant to tok stori. This can include information relevant to all three domains of the Melanesian mind as well as conflicts leaders have experienced between domains. As a result, both participants and mentors can explore lived experiences of leadership rather than a subset of experiences restricted by an uncertain understanding of the methodology in use. The effect is that:

Tok stori leadership creates confidence and trust and [is] not threatening [to] people's opinions.

Compared to the initial proposal for an outcome-focused semi-structured interview methodology, mentors understand a tok stori approach as producing a more balanced experience of power. This has the potential to create a space for trust where what a leader thinks is legitimized and thus more likely to be voiced.

\section{Question 2: Issues of effectiveness}

On the question of effectiveness, three aspects of effectiveness are discussed.

\section{Effectiveness indicated by results}

Mentors describe evaluation performed in-situ as contributing to the effectiveness of tok stori as a pedagogical tool. This suggests that the effectiveness of tok stori is a matter of participation and deep engagement. The pedagogical effects of such engagement may take time because tok stori is a process. However, to set the stage for effectiveness, the relational tenor of tok stori needs to be understood at the outset. According to one mentor,

Effectiveness is getting it right from the start; never mind how long it takes. Change is not immediate and instant but getting it right is paramount. Getting it right with school leaders, school leaders understanding it and being willing and open to implement; this is effectiveness.

Understanding which leads to change can develop through tok stori because the process allows participants to embed new ideas into existing complex multi-domain "lived" schema. This process is supported by trust and the way tok stori invites a comprehensive appreciation of experience. Being effective by getting it right is a relational matter. Effective pedagogical tok stori is indicated by ownership. Where there is "more tok by the participants (say above 85\%) then tok stori does take place". Mentoring encounters where the mentor feels constrained to gain certain responses by the demands of structure can undermine the ownership of leader-participants. Ultimately, in tok stori where deep relationality has developed, "people need to simply say to you, yea I know what you are talking about. That is effectiveness".

\section{Effectiveness as giving respect and building relationships}

Since to take part in tok stori is to inhabit a relational ontology, respectful relations are a key marker of effectiveness. Mentors understand the significance of trust in relationships for effective tok stori: 
In reflecting on our tok stori with Solomon Island educational leaders... it is very important to build a trusting relationship between educational leaders and researchers or mentors or helpers.

Mentors describe tok stori as a relationally enabling experience, a process where respect leads to trust which can inspire actions for change:

The tok stori has added value to our being with our people in a manner which accords respect to them as leaders. Doing so allows leaders to show respect to those whom they trust and would [be likely to] make change happen.

Mutually beneficial respectful relationships are intertwined with the kinds of reflection which can stimulate change. Where tok stori is embraced as a pedagogical tool, relationships can be configured so that leaders are "impowered" (Nakhid, 2003, p. 304), that is, invited into a space where the power they have over their own lives and understandings is acknowledged and fulfilled.

A further indicator of the effectiveness of tok stori is the emergence of rich data, capable of thick description (Geertz, 1973). An example of this is when trust leads to contextual interpretation of negative leadership experiences. Experienced mentors understand the effectiveness of tok stori in this regard by comparison to other methodologies:

You get more and richer information in tok stori than with interviewing. In addition to that, once you form a relationship of trust, you can advise or even they will easily share with you about their struggles.

This is not surprising if an interview is understood as "relationship between two people where both parties behave as though they are of equal status for its duration, whether or not this is actually so; and where, also, both behave as though their encounter had meaning only in relation to a good many other such encounters" (Benney \& Hughes, 1956, p. 142). Tok stori connects those involved more deeply, more authentically and without time limit. Consequently, despite its ubiquity, the stakes are higher. The consequence of deep relational investment is greater potential for pedagogically framed change.

\section{Effectiveness as creating a safe space}

Tok stori is a relational activity which takes place in time and space. The way relationships exist in time and the cultural inflections or nuances of the tok stori space distinguish it from many other pedagogical forms. A sense of safety built on relational trust can flow from relationally-focused storying which takes time and skill to develop and is dependent on a shared understanding of tok stori as a relevant form of pedagogical interaction.

[T]ok stori is a creation of space for people to tell their own stori. That created space is important because it is from there that an educational leader is to tell their stori.

Safety to reveal oneself is an essential element in telling one's story: conflicts between individuals, conflicting demands for loyalty from the domains of culture (kastom), church and formalized institutions, and the whole gamut of experience, positive and negative, are involved. The qualities of the tok stori space can be evaluated in-situ through sensitized, reflective mentoring. One mentor noted that: 
[T]he extent to which a leader shares about what is important to them depends very much on how the environment and climate for tok stori is created and built.

This places onus on leadership mentors to be well-versed in tok stori and have a secure grasp of an appropriate model of leadership. It demands that funders and programme organizers invest the time and cultural understandings required to support mentors in their endeavours.

\section{Question 3: Issues of reflection}

In answering this question, two issues: mindset and cultural data gathering, are discussed.

\section{Reflection on mindset}

Mentors are able to reflect on their own use of tok stori and the way advocacy for tok stori in leadership development affects them. This is unsurprising since tok stori is an interactive, relational process which aims to be mutually beneficial. The disturbance posed to previously held ideas about leadership development is a key benefit of aligning leadership practice with tok stori in Melanesia. One mentor explained this directly:

Up until my use of tok stori in leadership development, my own approach to improving leadership has been quite linear... This was an important realisation for me, that reality is not lineal. Reality is complex. Having understood this, we began to look for different ways of supporting leaders in their own contexts [so as] to be of help to them as mentors.

Tok stori, therefore, can have catalytic power in shifting the mindset of Melanesian mentors, harnessing the complex understanding of lived reality of the Melanesian mind, and encouraging access of culturally founded expertise. This is possible because tok stori

[Is] a way of thinking. It is a way of life. It is how we live, how we read, scrutinize and analyse whoever is asking us, questioning us, or campaigning for us.

Because it is ubiquitous in Melanesian life, tok stori, when admitted into non-traditional contexts by sensitive programme design, can reorganize conceptual as well as methodological matters.

Mentors gain from the experience of practicing tok stori in leadership development. This involves learning about the form through its placement in a new context, as well as experiencing the way time in tok stori redefines pedagogical relationships:

I have learned a lot using tok stori. It has broadened my personal perceptions about many things. One area is the contextual knowledges and experiences. Why? Because tok stori is a two-way process [which] happens simultaneously. The process is instant and responsive. It involves pausing, questioning to understand and reflecting on what is being shared.

This response suggests that mentors, conscious and deliberate in their participation in tok stori as pedagogy, can appreciate at increased depth the contextual nature of what is known as it has been experienced by those storying. This has the potential to increase mentor's future expertize in harnessing the pedagogical potential of tok stori. In addition, because the tok stori process requires the deep commitment of mentors in space and time, mentor engagement intensifies the relationality between leader and mentor. When this is 
the subject of reflection, more understanding about the processes involved can be gained by mentors.

\section{Reflection on cultural data gathering}

Mentors identify tok stori as an appropriate cultural tool for gathering data in three ways: legitimacy or ownership, comfort and depth. Firstly, legitimacy. One mentor explained a new understanding of tok stori as a legitimate research activity as a result of experiencing it in a pedagogical setting:

I appreciate tok stori more as a form of obtaining legitimate, people-owned data and information from our people in a medium they are comfortable with and [which] is part of their life and culture.

Naming tok stori as the basis of a leadership programme helps Melanesian leaders to identify tok stori as pedagogy as a process which they can own and use in leadership. This is because indications of legitimacy honour Melanesian culture and advocate for its value. Leadership development which ignores contextuality is unlikely to gain situationally-soaked maximally useful data or provide a path from leadership development into contextual leadership practice.

Mentors understand that tok stori as a pedagogical tool can enhance the levels of comfort experienced by leaders with the beneficial effect of providing rich data. For instance:

Tok stori empowers our people as it provides an opportunity for our people to provide data in the comfort of their own contexts (home, school, house, village etc). When our people are comfortable, they will share from their hearts.

When people are comfortable, they are likely to reveal the intricacies and difficulties of their lives. Comfort is linked to culture because it is a state of satisfaction promoted by a holistic understanding of a situation which offers the assurance that one's conduct and understanding are appropriate and appreciated. Discomfort, by contrast, can be created by a sense of uncertainty, confusion, unfamiliarity or relative powerlessness:

If questions were asked (as in semi-structured interviews) about sensitive topic areas, responses appear to be tardy. The tok stori approach, however, enabled a free-flowing conversation.

This contrast reiterates mentors' appreciation of the Indigenous approach of tok stori compared to an approach from non-Melanesian culture. Gathering information which has a cultural basis through a mode embedded in that culture is logical. This logic can promote full, comfortable participation.

Finally, leaders reflexively observed the depth of self-knowledge gained from involvement in tok stori as pedagogy. Where full participation takes place, self-awareness can result. This is important in a leadership model which sees the elements of leadership as including: the person as leader; their actions; their reference point or "True North"; and their development. Self-awareness is essential for leaders to appreciate the way personally-centred elements of leadership exist in culturally, cosmologically and institutionally defined contexts. Without self-awareness, new knowledge and experiences will remain as superficial learnings of limited use, rather than deep appreciations of the potential of the self to contribute to leadership: 
The tok stori approach also enabled, on the leader's part, a self-exploration of what they may have said on the surface but that which required further attention.

The processual nature of tok stori and leaders' levels of comfort as expert practitioners in tok stori have the potential to support continued self-investigation and ongoing leadership development.

In the next section, we restate the findings of the study and discuss their ontological and relational implications for donor-funded programmes in Melanesia.

\section{DISCUSSION}

Tok stori is an everyday reality in Melanesia; hence its relevance for Melanesian leadership development programmes. This relevance is at two levels. First, at a cultural level, tok stori is preferred in Melanesian leadership development contexts because it affirms leaders and respects them on their own terms. Additionally, tok stori enables Melanesian leaders to use their strengths in oral communication for their further leader development. Because Melanesian leaders already have well-developed oral skills, they can then focus on developing higher-level leadership capabilities using tok stori.

Second, at a personal level, tok stori is very relevant for Melanesian leaders. As stated, leaders use tok stori daily within family, school and in village settings. Leaders prioritize tok stori because of its relational value and its potential for enhancing shared purpose and mutual benefit. Because they are familiar with tok stori, leaders are encouraged to be themselves, remaining true to their own cultural identity. As themselves, they can share their lived joys and lived sorrows easily and trustingly with others.

The cultural relevance of tok stori for leadership development programmes in Melanesia diverges from often-used donor leadership development programme strategy, practice and research. Perhaps this mismatch is a crucial element in the recurring failure experienced in the post-training implementation of donor-devised approaches as lamented by Sanga (2009). It may well be that international donors and Pacific Islands stakeholders involved in leadership development programmes would benefit by extending their own boundaries to embrace tok stori leadership and pedagogy. Operationally, because tok stori is not yet commonly used in donor-funded leadership development programmes, the activity of donors and other stakeholders in adopting tok stori may support its theoretical and practical development in international development settings.

On the question of the effectiveness of tok stori pedagogy for Melanesian leadership development, we argue that tok stori is effective in three ways. First, tok stori permits deep engagement by tok stori actors. This enables Melanesian leaders to own their authentic leader development ideas and actions where these are derived from and related to their existing complex "lived" schema of leadership. Second, because tok stori inhabits a relational ontology, it is effective in fostering respectful relationships with Melanesian leaders. As a relationally enabling process, tok stori encourages trust to be built among tok stori actors, thereby stimulating further development change for leaders. Third, as a relational activity, tok stori is effective because it creates safe spaces where deep conversations can take place. Conversations of this nature enable deepening understandings to be reached between mentors and leaders. 
Numerous donor-supported leadership development programmes continue to take place throughout Melanesia. The delivery effectiveness of such programmes is often assumed and not demonstrated. When effectiveness is assessed, it is often measured in managerial and organisational terms. In the end, measures of effectiveness for leadership development programme pedagogy become matters of donor-focused politics, choice, epistemology and methodology. Given this study's findings, perhaps as yet unconsidered ways of effectiveness might be embraced. International development programme design might consider seeking effectiveness through tok stori so that it is reflective of the Melanesian mind. This kind of change requires a rethink, beginning with valuing the people-returns of programmes for Melanesia.

In examining how tok stori affects Melanesian mentors, we point to impacts at two levels. First, the application experience of tok stori in a donor-funded leadership development programme has shifted mentors' mindsets about and towards appreciating culturally founded knowledge, expertise, and pedagogy in international development programmes. In addition, mentors deemed their experience as valuable learning, capable of enhancing their future expertize by harnessing the pedagogical potential of tok stori.

Second, Melanesian mentors see tok stori in leadership development programmes as an appropriate cultural tool. Having applied tok stori as a research tool, mentors found tok stori to be legitimate and honouring of Melanesian culture. Tok stori is empowering to Melanesian leaders because it enhances their cultural comfort, which in turn leads them to greater openness in sharing their leadership in its intricacies and difficulties with mentors. Moreover, Melanesian mentors see the appropriateness of tok stori for its potential to increase the depth of self-knowledge and personal development.

An examination of donor-funded leadership programmes in Melanesia will show that these programmes do not capture the personal learning of mentors, programme trainers or developers. If personal learning is documented, often it is that of programme participants and not mentors. Given the valuable insights of the personal learning of Melanesian mentors, perhaps donors and other stakeholders might include researching mentor learning as an integral part of international development programmes. Indeed, perhaps inclusion of personal learning which engages our understanding of the Melanesian mind beyond managerial and organizational learnings can be explored in donor programmes. Ultimately, a desirable future is one where donors are engaging with the Melanesian mind in programme spaces.

\section{CONCLUSION}

The title of this article, "Melanesian Tok stori in leadership development: Ontological and relational implications for donor-funded programmes in the Western Pacific", suggests what is at stake in making culturally-mediated changes in leadership (and other) programmes, funded and otherwise, in Melanesia. Different people legitimately and inevitably have different world views, but issues which derive from ontology in intercultural contexts revolve around the qualities of relationships. The relationship between donors and programme recipients has been implicitly addressed through a consideration of what happens when the cultural wisdom of recipients is embraced in a donor-funded context. Well-meaning imported understandings which do not deliberately honour place-based thinking can draw accountability borders around programmes erasing much of what could count: already held, contextually developed expertize as a 
programme resource; the development of leadership across all those involved in a programme as an outcome; and learning about the world views of others as a contribution to inter-cultural understanding. Through tok stori, this article has demonstrated that the aims of donor-funded programmes, if suitably reframed and re-actioned, can be attained through Indigenous means. Where there is a lack of relational equity, the imposition of thought and action which reflect the ontology of donor-culture can result in a loss of potential gains for all. However, where those developing and enacting programmes inhabit a negotiating space (Mila-Schaaf \& Hudson, 2009) capable of appreciating and valuing ontological difference, the harnessing of everyday cultural practices offers a rich navigation in many donor-supported fields, including leadership development. This story of tok stori in Melanesia offers a vibrant example.

\section{APPRECIATIONS}

Appreciations are offered to the following FFM members who participated in this study: Joseph Pitakia, Jack Maebuta, Joash Maneipuri, (the late) Samson Rihuoha, Patterson Beuka, Jean Tafoa and Stanley Houma; all of the Solomon Islands.

\section{REFERENCES}

Akao, S. M. (2008). Seen but not heard: Women's experiences of educational leadership in Solomon Islands secondary schools. The University of Waikato. Retrieved from https://hdl.handle.net/10289/2379

Benney, M., \& Hughes, C. (1956). Of sociology and the interview: Editorial preface. American Journal of Sociology, 62, 137-142.

Brigg, M., Chadwick, W., Griggers, C., Murdock, J., \& Vienings, T. (2015). Solomon Island National Peace Council: Inter-communal mediation. Retrieved from https://espace.library.uq.edu.au/data/UQ_373816/brigg_et_al_2015_nat_peace_m edn.pdf

Evans, T., Guy, R., Honan, E., Paraide, P., \& Muspratt, S. (2010). Reflections on conducting educational research projects in Papua New Guinea. Journal of Research and Reflections in Education, 4(2), 72-89.

Fa'avae, D., Jones, A., \& Manu'atu, L. (2016). Talanoa 'i 'ae talanoa-talking about talanoa: Some dilemmas of a novice researcher. AlterNative: An International Journal of Indigenous Peoples, 12(2), 138-150.

Fernandez, S. (2016). Intersections between Pacific leadership and international development. Retrieved from URI: http://hdl.handle.net/10063/5036

Geertz, C. (1973). Thick description: Toward an interpretive theory of culture. Retrieved from https://philpapers.org/archive/GEETTD.pdf

George, W. (2007). True North: Discover your authentic leadership. Massachusetts: Centre for Businness Ethics. Retrieved from https://www.bentley.edu/sites/www.bentley.edu.centers/files/centers/cbe/georgemonograph.pdf 
George, W., Sims, P, Mclean, A., \& Mayer, D. (2007). Discovering your authentic leadership. Harvard Business Review. Retrieved from

https://www.simplylifeindia.com/uploads/8/1/1/5/8115412/discovering_your_auth entic_leadership.pdf

Halapua, S. (2000). Talanoa process: The case of Fiji. Retrieved from http://unpan1.un.org/intradoc/groups/public/documents/un/unpan022610.pdf

Houma, S. (2008). Learning from traditional leadership: A case study of 'Iki'oa Maitani'ohaoroha Chief and Priest. Retrieved from http://www.governance.usp.ac.fj

Houma, S. (2011). Leadership challenges and management strategies of women school leaders. In G. I. Lingam (Ed.), Educational leadership: Emerging issues and successful practices (pp. 139-153). Suva, Fiji: University of the South Pacific.

Malasa, Donald. (2007). Effective school leadership: An exploration of the issues inhibiting the effectiveness of school leadership in Solomon Islands' secondary schools (Thesis, Master of Educational Leadership (MEdLeadership)). The University of Waikato, Hamilton, New Zealand. Retrieved from https://hdl.handle.net/10289/2429

Mila-Schaaf, K., \& Hudson, M. (2009). Negotiating space for indigenous theorising in Pacific mental health and addictions. Retrieved from http://www.tepou.co.nz/uploads/files/resource-assets/negotiating-space-forindigenous-theorising-in-pacific-mental-health-and-addictions.pdf

Nakhid, C. (2003). "Intercultural" perceptions, academic achievement, and the identifying process of Pacific Islands students in New Zealand schools. The Journal of Negro Education, 72(3), 297-317. doi:10.2307/3211249

Nanau, G. L. (2014). Local experiences with mining royalties, company and the state in the Solomon Islands. Journal de la Société des Océanistes 138-139, 77-92.

Sanga, K. (2009). The Pacific public servant: Serving three masters? Paper presented at the Graduate School of Public Policy, University of Saskatchewan, Canada.

Sanga, K. (2016). What if form was the aid? Possible opportunities for renewed neighbourliness in aid relationships. International Education Journal: Comparative Perspectives, 15(3), 7-15.

Sanga, K. (2017). A facilitation "guide” for leaderful tok stori. Unpublished manuscript.

Sanga, K., \& Walker, K. D. (2005). Apem Moa: Solomon Islands leadership: He Parekereke, Institute for Research and Development in Maori and Pacific Education, Victoria University.

Sisiolo, Lenga. (2010). "Being" an educational leader in Choiseul Province in the Solomon Islands: A case study of the context of leadership for principals and deputy principals. (Thesis, Master of Educational Leadership (MEdLeadership)). The University of Waikato, Hamilton, New Zealand. Retrieved from https://hdl.handle.net/10289/4411 
Spence, R., \& Wielders, I. (2006). Conflict prevention in the Pacific. Retrieved from Canberra, Australia:

Talanoa \& Development Project. (2005). Tok Stori. Retrieved from http://talanoa.org/TDP_Solomon_Islands_Tok_Stori.html

Vaioleti, T. (2013). Talanoa: Differentiating the talanoa research methodology from phenomenology, narrative, Kaupapa Maori and feminist methodologies. Te Reo, $56,191-212$.

Van Heekeren, D. (2014). Naming, mnemonics, and the poetics of knowing in Vula'a oral traditions. Oceania, 84(2), 169-184.

Vella, L., \& Maebuta, J. B. (2018). Building national unity, reconciliation and peace in the Solomon Islands: The missing link. In B. Jenkins, D. B. Subedi, \& K. Jenkins (Eds.), Reconciliation in conflict-affected communities: Practices and insights from the Asia-Pacific (pp. 57-74). Singapore: Springer Nature.

Yosso, T. J. (2005). Whose culture has capital? A critical race theory discussion of community cultural wealth. Race Ethnicity and Education, 8(1), 69-91. doi:10.1080/1361332052000341006 